\title{
OneLab Tutorial: A Single Portal to Heterogeneous Testbeds
}

\author{
Loic Baron, Radomir Klacza, Mohammed Yasin Rahman, Ciro Scognamiglio, Nina Kurose, \\ Timur Friedman, Serge Fdida \\ UPMC Sorbonne Universités \\ 4 Place Jussieu \\ 75005 Paris, France \\ firstname.lastname@lip6.fr
}

\section{MOTIVATION}

Large-scale experimentation in varying types of environments is difficult to achieve, and until recently, experimenters have had to either construct their own platforms or rely on simulation, emulation, or analysis to gain results. However, these methods largely do not provide adequate or verified results. There lacked a viable model for the federation of large-scale testbeds that reconciled the challenges posed by how to provide a single entry point to access heterogeneous and distributed resources, and how to federate these resources that are under the control of multiple authorities. Efforts such as the FIRE ${ }^{1}$ initiative in Europe and $\mathrm{GENI}^{2}$ in the United States have worked to develop such a model, and the OneLab ${ }^{3}$ experimental facility, which came online in 2014, realizes this model, making a set of world-class testbeds freely available to researchers through a unique credential for each user and a common set of tools. OneLab provides a largescale facility for rapid and remote testing that produces exhaustive results, at no charge to the experimenter. We allow users to deploy innovative experiments across our federated platforms that include the embedded object testbeds of FIT IoT-Lab ${ }^{4}$, the cognitive radio testbed of FIT CorteXlab ${ }^{5}$, the wireless testbeds of NITOS-Lab ${ }^{6}$, and the internet overlay testbeds of PlanetLab Europe (PLE) ${ }^{7}$, which together provide thousands of nodes for experimentation. The OneLab portal allows single-entry point access to these platforms, and provides users with unique credentials. This is made possible through the adoption of Slicebased Federation Architecture (SFA), an API for authentication and authorization that was conceptualized by the GENI initiative in the US, where each authority authenticates users and authorizes access to the resources; and MySlice ${ }^{8}$, the portal technology that we have developed to federate heterogeneous resources.

\section{AIMS}

The focus of our tutorial will be on providing attendees with hands-on experience in navigating the OneLab portal and

\footnotetext{
${ }^{1}$ http://www.ict-fire.eu/home.html.

${ }^{2}$ http://www.geni.net/.

3 S. Fdida, T. Friedman, T. Parmentelat. "OneLab: An Open Federated Facility for Experimentally Driven Future Internet Research". In Tania Tronco, ed., New Network Architectures: The Path to the Future Internet, Studies in Computational Intelligence. Springer Verlag, vol. 297, pp. 141-152, 2010; http://onelab.eu/.

${ }^{4}$ http://www.iot-lab.info/.

${ }^{5}$ http://www.cortexlab.fr/.

${ }^{6} \mathrm{http}: / /$ fit-nitos.fr/.

${ }^{7}$ http://planet-lab.eu/.

${ }^{8} \mathrm{http}: / /$ www.myslice.info/
}

deploying experiments across our federated platforms. We will open with a short explanation of our experimental facility, its testbeds, and the ways in which OneLab reconciled the technical challenges of federating: how SFA allows for the federation of resources under multiple authorities and how MySlice provides a user-friendly interface displaying resources in web format. Following this overview, we will show attendees how to deploy an experiment on one of our FIT IoT-Lab testbeds through the OneLab portal. The projected outcome of this tutorial is that through its completion, users will finish with an account on the OneLab portal and the knowledge on how to access and deploy experiments on dozens of world-class testbeds.

The tutorial will walk the attendees through the process of creating an account, reserving resources, accessing the gateway server and running a basic experiment from the console. Users will be able to sign up and we will authorize their accounts on the spot, so that they may immediately access portal resources and deploy the experiments described below. We will launch three basic experiments on three of our different platforms: FIT IoTLab, NITOS, and PLE; so as to demonstrate the entire process of testing through the portal.

The first experiment on FIT IoT-Lab will teach participants how to navigate the testbed tools. They will deploy their experiment, and then interact with running nodes by reading sensor values and sending radio packets. The purpose of this experiment will be to allow all attendees to have an introductory experience on navigating the OneLab portal and reserving resources, as they will both book IoT-Lab resources and view results remotely.

The second experiment will allow users to reserve NITOS nodes and use the OMF (cOntrol and Management Framework) framework to control the experiment. This will give users the opportunity to learn how to use the OMF tool for experiment management and control.

Lastly, the experiment on PLE will provide each user with various initscripts describing different experiments. They will use these to configure their reserved PLE resources, deploy experiments, and view the output of each experiment. Users who are already familiar with PlanetLab will see how to deploy experiments through the new polyvalent OneLab platform.

\subsection{Target Audience}

Our tutorial is targeted towards all users, coming from beginner to advanced backgrounds in experimental platforms. The OneLab facility is completely open to researchers, students, and scientific, academic, or industrial innovators. Those who are interested in IoT testing, wireless testing, or testing on internet-overlaid 
platforms will all take a particular interest in our tutorial experiments, as will those interested in learning OMF as a tool. The IoT-Lab experiments will particularly interest those dedicated to the WSN community, and especially to people who are looking for an accurate scientific tool to support the design, development, tuning, and experimentation of real large-scale sensor network applications. All of our platforms are generic, open and flexible, meaning that a user can deploy his or her applications without any kind of restrictions on the programming language, the programming model, or the OS. It may be used by people working on the physical layer, the MAC layer, or any other layer of the OSI model, or on a more global application.

\section{DURATION AND SPEAKERS}

The tutorial session will last two hours. A list of speakers is as follows:

Loic Baron, the lead engineer of the OneLab team, will present the OneLab federation and guide the participants through using the portal. He will be assisted by an engineer from FIT IoT-Lab to help in conducting the hands-on tutorial. 